\title{
A SVD-Based Optical MIMO Precoding Scheme in Indoor Visible Light Communication
}

\author{
Qing-Feng Liu, Shuai-Fang Xiao, Kai-Zhi Huang, and Zhou Zhong
}

\begin{abstract}
A novel optical precoding scheme based on singular value decomposition (SVD) is proposed to solve the problem that strong channel correlation causes low spatial multiplexing gain in indoor visible light MIMO communication. This scheme first ensures a nonnegative signal after precoding through mapping the transmit bits into a specific symbol constellation. Then it eliminates the inter-channel interference by decomposing the MIMO channel through SVD. Finally, it reduces the BER by optimally allocating power among the sub-channels with the object to make the condition of each sub-channel equal. The simulation results show that SVD-based optical precoding scheme can significantly reduce the BER compared to $\mathrm{ZF}$ algorithm. And with the enhancement of channel correlation, the BER performance remains in a relatively low level, so it can obtain high spatial multiplexing gain under the strong correlation channel.
\end{abstract}

Index Terms-Indoor visible light communication, optical MIMO, precoding, SVD, power allocation.

\section{INTRODUCTION}

Visible Light Communication (VLC) is a novel kind of communication technology which is able to achieve high speed data transmission in indoor communication. Thus, VLC attracts extensive attentions worldwide. The optical wavelengths of light is used as the carrier of information in VLC, and the Light Emitting Diodes (LEDs) emit high speed flicker optical signals to transmit information, while the Photodetector (PD) or other optoelectronic transform devices receive the modulated optical signal and transform it to current signal. Compared to the traditional radio frequency communication and other optical communications, VLC has many advantages such as high transmitted power, non-electromagnetic interference, safety, and green. From what has discussed above, we can draw the conclusion that VLC has a great prospect for development [1]-[3].

The goal of indoor VLC is to achieve high speed data transmission [4], but the modulation bandwidth of white LED is just about $20 \mathrm{MHz}$ [5], which is too limited to realize high speed data transmission in Single Input Single Output (SISO) system. When Multiple Input Multiple Output (MIMO) technique is applied in indoor VLC, it could

Manuscript received January 20, 2014; revised May 13, 2014. This work was supported by National High Technology Research and Development Program of China (No. 2013AA013603), and Open Research Fund of State Key Laboratory of Mobile Communications (No. 2013D09).

Shuai-Fang Xiao is with the National Digital Switching System Engineering \& Technological Research Center, Zhengzhou 450002, China (e-mail: xiaoshuaifang@gmail.com).

Qing-Feng Liu, Kai-Zhi Huang, and Zhou Zhong are with the National Digital Switching System Engineering \& Technological Research Center, Zhengzhou 450002, China. potentially increase the scope of the communication link, overcome the interruption which is caused by personnel walks or shadows of the furniture in the room, and improve the reliability of the link. Meanwhile, the spectrum efficiency can be increased without the enhancement of bandwidth or transmit power [6], and the high data transmission will be achieved. In addition, indoor illumination needs many white LEDs, which provide rich spatial resources for VLC. Free space optical MIMO is first researched by Wilson in [7]. Indoor VLC MIMO system is first applied by O'Brien in [8]-[10], where a non-imaging receiving system and a imaging receiving system are introduced. And the experiment verifies that the indoor VLC MIMO system can obtain high spatial multiplexing gain. The performance of Repetition Coding (RC), Spatial Multiplexing (SMP) and Spatial Modulation (SM) are analyzed and compared in [11]. While the literature [8] and [11] indicate that indoor VLC MIMO channels have a strong correlation because of the Intensity Modulation (IM) and Direct Detection (DD), which leads to the lack of frequency and phase components. So it is difficult to obtain high spatial multiplexing gain, and the data transmission can't be fast enough. Literature [12] proposes a modulation scheme of VLC based on precoding matrix , but it doesn't consider the correlation of MIMO channels. How to obtain high spatial multiplexing gain and better use the rich spatial resources of indoor VLC is still a hot issue.

In order to solve this problem, this paper proposes an indoor optical precoding scheme based on Singular Value Decomposition (SVD), we call it SVD-based Precoding. First, the information transmitted must be mapped from a specific symbols constellation to ensure that the signals are nonnegative after precoding. Then, we decompose the indoor VLC MIMO channels by SVD, which can obtain the independent sub-channels to eliminate inter-channel interference. Finally, we solve the optimization problem aiming at the minimum system BER and subjecting to the limited total power to get the optimal power allocation matrix, and allocate the power among the sub-channels according to the matrix to reach the equal performance of each sub-channel, which can decrease the system BER. The receiver employs Maximum Likelihood (ML) algorithm to decode the received symbols after shaping process, and recovers the information by inverse symbols mapping. The system BER simulation results indicate that the BER of proposed SVD-based Precoding is significantly lower than ZF algorithm under different correlation channel. And with the enhancement of channel correlation, the BER performance remains in a relatively low level, so it can obtain high spatial multiplexing gain under the strong correlation channels. 


\section{SYSTEM MODEL}

In our VLC MIMO system, the number of white LED and PD is $N$ and $M$, respectively, where $M \geq N$. Using the serial-to-parallel converter, the information bits are transmitted through the white LEDs. The transmitted signal vector is denoted by $s=\left(s_{1}, \cdots, s_{i}, \cdots, s_{N}\right)^{T}$, where $1 \leq i \leq N$. $\mathbf{s}$ go through the indoor VLC channel, and finally reach the receiver. At the receiver, PDs turn the optical power signal into current signal, and then add the white Gauss noise signal $n$, where $\boldsymbol{n}=\left(n_{1}, \cdots, n_{j}, \cdots, n_{M}\right)^{T}, 1 \leq j \leq M$. Thus we get the output current signal $\boldsymbol{y}=\left(y_{1}, \cdots, y_{j}, \cdots, y_{M}\right)^{T} \cdot y_{j}$ is the output current signal of the $j$ th PD which is given by:

$$
y_{j}=r \sum_{i=1}^{N} h_{j i} s_{i}+n_{j}
$$

where $r$ is the photoelectric conversion factor, $h_{j i}$ is the Direct Current (DC) channel gain from the $i$ th white LED to the $j$ th PD. Thus, the matrix is represented as:

$$
\boldsymbol{y}=r \boldsymbol{H} \boldsymbol{s}+\boldsymbol{n}
$$

where $H$ matrix is the DC channel gain, and the dimension is $M \times N$.

In the indoor environment, light signals generated by the white LED lamp reach the receiver through direct path and diffuse path. Through the experiment, literature [13] finds that more than $90 \%$ of the optical signal power at the receiver comes from the direct component, and that from the diffuse component can be ignored. So we consider only the direct path here. We use Lambert radiant model to describe the luminescence mechanism of white LED light. The Lambert radiant intensity can be expressed as:

$$
R_{0}(\phi)=[(m+1) / 2 \pi] \cos ^{m} \phi
$$

where $\phi$ is the emission angle, $m$ is the order of Lambert emission. $m=-\ln 2 / \ln \left(\cos \psi_{1 / 2}\right), \psi_{1 / 2}$ is the half power emission angle. Thus the DC channel gain from the $i$ th white LED to the $j$ th PD can be expressed as:

$$
h_{j i}=\left\{\begin{array}{cc}
\frac{A}{d_{j i}^{2}} R_{0}\left(\phi_{j i}\right) T_{f}\left(\varphi_{j i}\right) g\left(\varphi_{j i}\right) \cos \left(\varphi_{j i}\right), & 0 \leq \varphi_{j i} \leq \varphi_{c} \\
0 \quad, & \varphi_{j i}>\varphi_{c}
\end{array}\right.
$$

where $A$ is the collection area for the $j$ th receiver, $d_{j i}$ is the distance between the $i$ th LED and the ${ }^{j}$ th receiver, $\phi_{j i}$ is the emission angle, $\varphi_{j i}$ is the angle of incidence of the light at the receiver, $\varphi_{c}$ is the Field-of-view (FOV) of the receiver, $T_{f}\left(\varphi_{j i}\right)$ is the gain of the filter, $g\left(\varphi_{j i}\right)$ is the gain of the optical concentrator.

From Equation (4), the indoor VLC MIMO channel is deterministic, lacks of frequency and phase components, and suffers serious interferences among sub-channels. All these above lead to strong channel correlation, and then limit the transmission rate of the system. To solve this problem, SVD precoding technology is applied to indoor VLC MIMO systems in our article. We decompose the MIMO channel into several independent parallel sub-channels through SVD, and allocate more power in the sub-channels which have smaller DC channel gain. In this way, the SNR of these weak sub-channels is improved, and finally the BER performance of the entire system.

\section{SVD-Based Optical Precoding Scheme}

This section describes the SVD-based optical precoding scheme in indoor VLC MIMO system. First, we map the transmit bits into a specific symbol constellation at the transmitter. After that, we decompose MIMO channel through SVD, and allocate power among the sub-channels with the object that minimize the BER performance. Finally, the signal at the receiver will pass through the shaping process, and the transmitted signal could be recovered by ML decoding algorithm.

\section{A. Precoding Scheme at the Transmitter}

As indoor VLC adopts IM modulation, the transmitted signal must be non-negative, that is

$$
s_{i} \geq 0, i=1,2, \cdots, R_{H}
$$

where $R_{H}$ represents the rank of $\boldsymbol{H}$, and $R_{H} \leq \min (N, M)$. To ensure the transmission signal $\mathbf{s}$ become non-negative, the symbol constellation of $\mathbf{s}$ should be determined firstly. Considering the common used OOK modulation in indoor VLC system, the symbol constellation of $\mathbf{s}$ is taken as

$$
\boldsymbol{\Theta}=\left\{\boldsymbol{a}_{1}, \boldsymbol{a}_{2}, \cdots, \boldsymbol{a}_{L}\right\}
$$

where $L=2^{R_{H}}, \quad \boldsymbol{a}_{l}$ is a $N \times 1$ dimensional vector, and satisfies $\boldsymbol{a}_{l}=\left(a_{l, 1}, a_{l, 2}, \cdots, a_{l, R_{H}}, 0, \cdots, 0\right)^{T}, l=1,2, \cdots, L$, and the value of $a_{l, n}, n=1,2, \cdots, R_{H}$, is 0 or 1 . Assume $\boldsymbol{x}$ is the signal to be transmitted. To ensure that the transmitted signal is in the symbol constellation $\boldsymbol{\Theta}$, the symbol constellation of $\boldsymbol{x}$ is taken as

$$
\boldsymbol{\Xi}=V^{H} \Theta=\left\{\boldsymbol{b}_{1}, \boldsymbol{b}_{2}, \cdots, \boldsymbol{b}_{L}\right\}
$$

where $\boldsymbol{b}_{l}$ is a $N \times 1$ dimensional vector, and $\boldsymbol{b}_{l}=\left(b_{l, 1}, b_{l, 2}, \cdots, b_{l, R_{H}}, 0, \cdots, 0\right)^{T}, l=1,2, \cdots, L$. As $\boldsymbol{V}$ is a unitary matrix, signal $\boldsymbol{x}$ can be transformed into $\mathbf{s}$ in symbol constellation $\boldsymbol{\Theta}$ after precoding by the $\boldsymbol{V}$ matrix.

Assum $\boldsymbol{H}$ is known to the transmitter and receiver, then perform SVD decomposition on $\boldsymbol{H}$ : 


$$
\boldsymbol{H}=\boldsymbol{U} \boldsymbol{\Sigma} \boldsymbol{V}^{H}
$$

where $(\bullet)^{H}$ represents conjugate transpose, $\boldsymbol{V}$ is a $N \times N$ dimensional unitary matrix, $\Sigma$ is a $M \times N$ dimensional diagonal matrix:

$$
\Sigma=\left[\begin{array}{llll}
\sigma_{1} & & & \boldsymbol{O} \\
& \ddots & & \\
& & \sigma_{R_{H}} & \\
\boldsymbol{O} & & & \boldsymbol{O}
\end{array}\right]
$$

where $\sigma_{i}\left(i=1,2, \cdots, R_{H}\right)$ is the $i$ th largest singular value. As indoor VLC MIMO channel has a strong correlation, the condition number cond $(\boldsymbol{H})$ of DC channel gain matrix $\boldsymbol{H}$ is very large, that is

$$
\sigma_{1} \gg \sigma_{R_{H}}
$$

Therefore, when using SMP technology, sub-channels with smaller singular value have poor channel conditions. When using the average power allocation, the SNR of these sub-channels are lower, and resulting in a higher system BER. In order to reduce the BER of the whole indoor VLC MIMO system, when designing the power allocation matrix, more power can be allocated on the sub-channels with smaller singular values to improve the received SNR. $P_{0}$ is the total transmit power, and the allocated power of different sub-channels is

$$
P_{i}=\rho_{i} P_{0} ; 0<\rho_{i}<1 ; i=1, \cdots, R_{H}
$$

Set $\sigma^{2}$ as the noise variance of each sub-channel, and then the receiving SNR of the $i$ th sub-channel can be expressed as

$$
S N R_{i}=\frac{\left(r \rho_{i} P_{0} \sigma_{i}\right)^{2}}{\sigma^{2}}=\frac{r^{2} P_{0}^{2}}{\sigma^{2}} \rho_{i}^{2} \sigma_{i}^{2}
$$

So the system BER with OOK modulation can be expressed as

$$
B E R=\frac{1}{R_{H}} \sum_{i=1}^{R_{H}} Q\left(\frac{r P_{0}}{\sigma} \rho_{i} \sigma_{i}\right)
$$

where the function $Q$ is

$$
Q(x)=\int_{x}^{\infty} \frac{1}{\sqrt{2 \pi}} e^{-y^{2} / 2} d y
$$

In order to obtain the optimal power allocation matrix, the following constraint equations is established

$$
\begin{array}{ll}
\text { minimize } & B E R \\
\text { subject to } & \sum_{i} \rho_{i}=1 \\
\text { known } & \sigma_{i}
\end{array}
$$

As the $Q$ is monotonically decreasing, the following equation must be satisfied to get the minimum BER

$$
\frac{r P_{0}}{\sigma} \rho_{i} \sigma_{i}=\frac{r P_{0}}{\sigma} \rho_{j} \sigma_{j} ; i \neq j
$$

Combining (16) with (15), we get

$$
\rho_{i, o p t}=\frac{1}{\sigma_{i} \sum_{j=1}^{R_{H}} \frac{1}{\sigma_{j}}}
$$

Thus the optimal power allocation matrix under minimum system BER criterion is

$$
\boldsymbol{\Lambda}_{o p t}=\left[\begin{array}{cccc}
\rho_{1, o p t} & & & \boldsymbol{O} \\
& \ddots & & \\
& & \rho_{R_{H}, o p t} & \\
\boldsymbol{O} & & & \boldsymbol{O}
\end{array}\right]
$$

And the optimal precoding matrix which meets the minimum system BER criteria can be expressed as

$$
\boldsymbol{F}_{o p t}=\boldsymbol{V} \boldsymbol{\Lambda}_{o p t}
$$

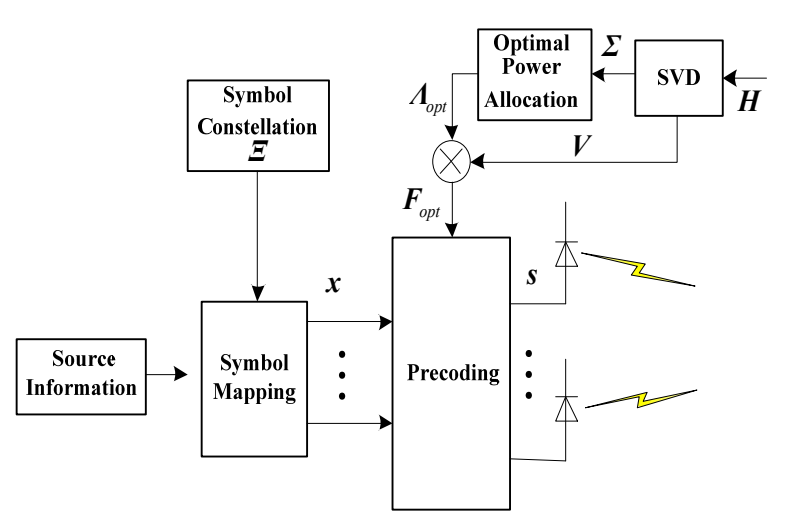

Fig. 1. Transmission processing flow of SVD-based optical precoding scheme in indoor VLC MIMO system.

The whole processing flow of the transmission scheme is shown in Fig. 1. Steps are as follows:

- Mapping each $R_{H}$ bits transmitted information into signal $\boldsymbol{x}$ in symbol constellation $\boldsymbol{\Xi}$.

- Perform SVD decomposition on $\boldsymbol{H}$, and get the unitary matrix $\boldsymbol{V}$ and sub-channel gain matrix $\Sigma$.

- According to $\Sigma$, solve the optimization sub-channel power allocation matrix $\Lambda_{o p t}$ with the target of minimizing the system BER.

- Using $\boldsymbol{V}$ and $\Lambda_{o p t}$ to calculate the optimal precoding matrix $F_{o p t}$.

- Preprocess the signal $\boldsymbol{x}$ by $F_{\text {opt }}$, and get the transmission signal $\boldsymbol{s}$.

\section{B. Decoding Scheme at the Receiver}

At the receiver, we use unitary matrix $U$ to shape the received signal $y$, and get $y$ 


$$
\begin{aligned}
\tilde{\boldsymbol{y}} & =U^{H} y \\
& =\Delta \boldsymbol{x}+\tilde{\boldsymbol{n}}
\end{aligned}
$$

where

$$
\Delta=\Sigma \Lambda_{o p t}=\left[\begin{array}{cccc}
\rho_{1, o p t} \sigma_{1} & & & \\
& \ddots & & \\
& & \rho_{R_{H}, o p t} \sigma_{R_{H}} & \\
\boldsymbol{o} & & & \boldsymbol{o}
\end{array}\right]
$$

Then we can recover the signal to be transmitted by $\mathrm{ML}$ decoding algorithm, and get

$$
\tilde{\boldsymbol{x}}=\arg \min _{l}\left\|\tilde{\boldsymbol{y}}-r \tilde{\boldsymbol{\Sigma}} \boldsymbol{b}_{l}\right\|_{F}^{2}
$$

where

$$
\begin{aligned}
\tilde{\boldsymbol{\Sigma}} & =U^{H} H F_{o p t} \\
& =\boldsymbol{\Sigma} \boldsymbol{\Lambda}_{\text {opt }}
\end{aligned}
$$

Finally, the messages can be recovered through the inverse symbol mapping of $\tilde{x}$. The whole processing flow at the receiver is shown in Fig. 2. Steps are as follows:

- Perform SVD decomposition on $H$, get the unitary matrix $U$ and $V$, and the sub-channel gain matrix $\Sigma$.

- According to $\Sigma$, solve the optimization sub-channel power allocation matrix $\Lambda_{o p t}$ with the target of minimizing the system BER.

- Using $\Sigma$ and $\Lambda_{o p t}$ to calculate optimized sub-channel gain matrix $\tilde{\boldsymbol{\Sigma}}$.

- Shape the received signal $y$ by unitary matrix $U$, and get signal $\tilde{y}$.

- Recover $\tilde{x}$ in constellation $\boldsymbol{\Xi}$ by means of $\mathrm{ML}$ decoding algorithm.

- Recover the source information through the inverse symbol mapping of $\tilde{x}$.

\section{System Simulation Results}

The channel correlation of MIMO channels in indoor VLC is related to the distance between LEDs and PDs, when $d_{h}$ is fixed, the smaller $d_{L E D}$ and $d_{P D}$ is, the stronger channel correlation and the greater the condition numbers of $H$ will be. The BER performance of the system with different channel correlation can be analyzed by changing $d_{L E D}$ or $d_{P D}$ to get $\boldsymbol{H}$ with different condition numbers. For $2 \times 2 \mathrm{MIMO}$ and 4 $\times 4$ MIMO scenarios with different distance spacing of LEDs and PDs, Monte Carlo simulation is employed to simulate the BER performance of the proposed SVD-based optical precoding scheme of indoor VLC MIMO system. MIMO spatial multiplexing is employed for the simulating systems. The spatial distribution of $2 \times 2$ MIMO and $4 \times 4$ MIMO scenarios are shown in Fig. 3 and Fig. 4, the simulation parameters are shown in Table I, the configuration of parameters derives from the literature [15].

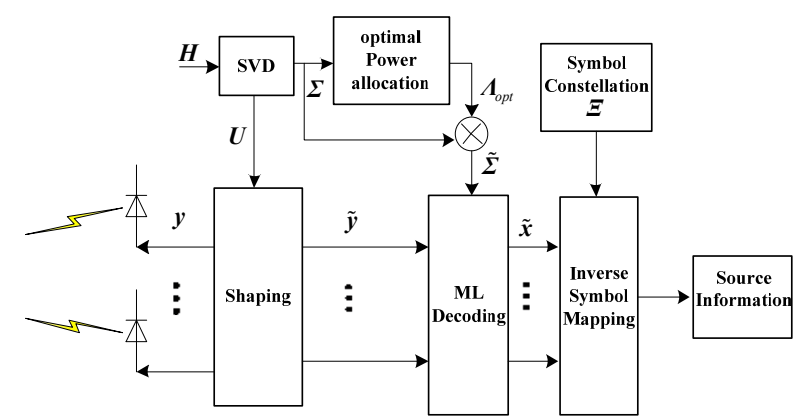

Fig. 2. Receiving processing flow of SVD-based optical precoding scheme in indoor VLC MIMO system.

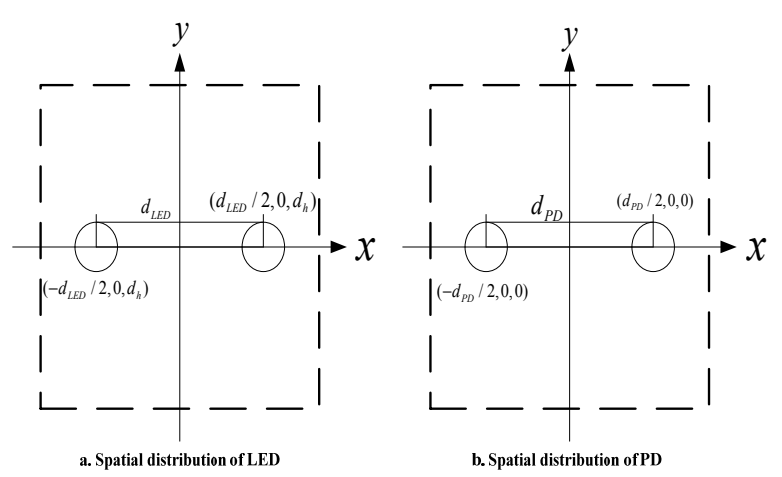

Fig. 3. Spatial distribution for indoor VLC $2 \times 2$ MIMO system.

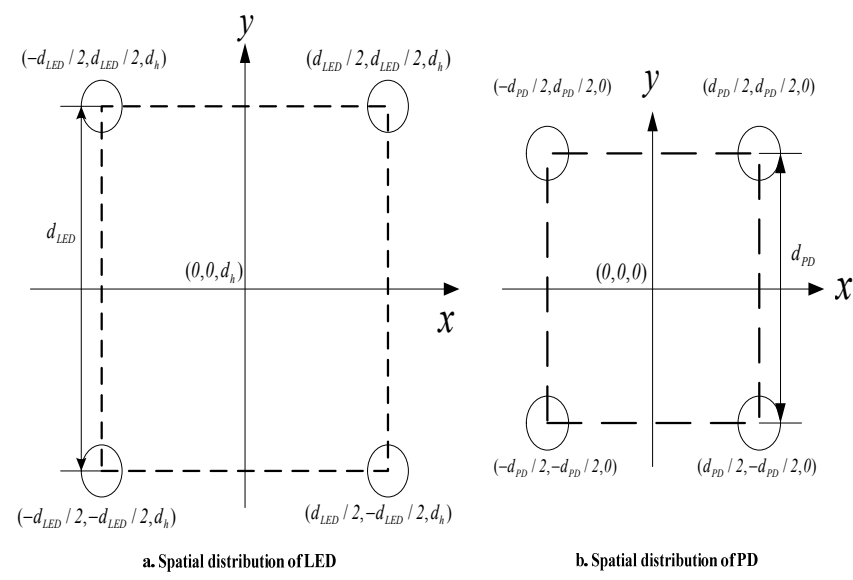

Fig. 4. Spatial distribution for indoor VLC $4 \times 4$ MIMO system.

\begin{tabular}{cccc}
\multicolumn{4}{c}{ TABLE I: SiMULATION PARAMETERS } \\
\hline \hline Parameters & Values & Parameters & Values \\
\hline Room Size & $5 \mathrm{~m} \times 5 \mathrm{~m} \times 3 \mathrm{~m}$ & $d_{h}$ & $2.25 \mathrm{~m}$ \\
$T(\psi)$ & 1 & $r$ & $0.4 \mathrm{~A} / \mathrm{W}$ \\
PD FOV & $60^{\circ}$ & $g(\psi)$ & 1.5 \\
$\Psi_{1 / 2}$ & $70^{\circ}$ & $\mathrm{A}$ & $1.0 \mathrm{~cm}^{2}$ \\
\hline \hline
\end{tabular}

Setting $d_{L E D}=0.8 m, d_{P D}=0.8 m$, then the DC channel gain matrix under $2 \times 2$ MIMO and $4 \times 4$ MIMO scenarios are described as follows:

$$
\begin{gathered}
\boldsymbol{H}_{2}=10^{-5} \times\left[\begin{array}{lll}
0.7762 & 0.6248 \\
0.6248 & 0.7762
\end{array}\right] \\
\boldsymbol{H}_{4}=10^{-5} \times\left[\begin{array}{llll}
0.7762 & 0.6248 & 0.5147 & 0.6248 \\
0.6248 & 0.7762 & 0.6248 & 0.5147 \\
0.5147 & 0.6248 & 0.7762 & 0.6248 \\
0.6248 & 0.5147 & 0.6248 & 0.7762
\end{array}\right]
\end{gathered}
$$

The condition numbers of the DC channel gain matrix are 
described as $\operatorname{cond}\left(\boldsymbol{H}_{2}\right)=9.2518$ and $\operatorname{cond}\left(\boldsymbol{H}_{4}\right)=61.514$, and in this case the correlation of MIMO channel is relatively weak, and the correlation of $4 \times 4$ MIMO channel is stronger than that in $2 \times 2$ MIMO scenario.

When $d_{L E D}=0.4 m$ and $d_{P D}=0.4 m$, the DC channel gain matrix under $2 \times 2 \mathrm{MIMO}$ and $4 \times 4$ MIMO scenarios are described as follows:

$$
\begin{gathered}
\boldsymbol{H}_{2}=10^{-5} \times\left[\begin{array}{lll}
0.7762 & 0.7334 \\
0.7334 & 0.7762
\end{array}\right] \\
\boldsymbol{H}_{4}=10^{-5} \times\left[\begin{array}{llll}
0.7762 & 0.7334 & 0.6942 & 0.7334 \\
0.7334 & 0.7762 & 0.7334 & 0.6942 \\
0.6942 & 0.7334 & 0.7762 & 0.7334 \\
0.7334 & 0.6942 & 0.7334 & 0.7762
\end{array}\right]
\end{gathered}
$$

The condition numbers of the DC channel gain matrix are described as cond $\left(\boldsymbol{H}_{2}\right)=35.267$ and $\operatorname{cond}\left(\boldsymbol{H}_{4}\right)=827.88$, and in this case the correlation of MIMO channel is relatively strong.

We further reduce $d_{L E D}$ and $d_{P D}$, setting $d_{L E D}=0.2 \mathrm{~m}$ and $d_{P D}=0.2 \mathrm{~m}$, and then the $\mathrm{DC}$ channel gain matrix under $2 \times 2 \mathrm{MIMO}$ and $4 \times 4 \mathrm{MIMO}$ scenarios are described as follows:

$$
\begin{gathered}
\boldsymbol{H}_{2}=10^{-5} \times\left[\begin{array}{lll}
0.7762 & 0.7652 \\
0.7652 & 0.7762
\end{array}\right] \\
\boldsymbol{H}_{4}=10^{-5} \times\left[\begin{array}{llll}
0.7762 & 0.7652 & 0.7544 & 0.7652 \\
0.7652 & 0.7762 & 0.7652 & 0.7544 \\
0.7544 & 0.7652 & 0.7762 & 0.7652 \\
0.7652 & 0.7544 & 0.7652 & 0.7762
\end{array}\right]
\end{gathered}
$$

The condition numbers of the DC channel gain matrix are described as cond $\left(\boldsymbol{H}_{2}\right)=139.40$ and $\operatorname{cond}\left(\boldsymbol{H}_{4}\right)=12647$, and in this case the correlation of MIMO channels is very strong.

We simulate the BER performance of the proposed SVD-based optical precoding scheme and compare it with the $\mathrm{ZF}$ algorithm under $2 \times 2 \mathrm{MIMO}$ and $4 \times 4 \mathrm{MIMO}$ scenarios with different spacing of LEDs and PDs. The simulation results are shown in Fig. 5. The BER curve of $2 \times 2$ MIMO scenario coincides with that of $4 \times 4$ MIMO scenario based on the proposed SVD-based optical precoding scheme in this paper, and what's more they are both significantly lower than MIMO systems employing the ZF algorithm, this is because the proposed SVD-based optical precoding scheme makes the performance of all sub-channels become similar by optimal power allocation process. Under the $2 \times 2$ MIMO scenario, the proposed SVD-based optical precoding scheme in this paper can achieve $15 \mathrm{~dB} \sim 40 \mathrm{~dB}$ SNR gain than ZF algorithm when the BER of the system is $2 \times 10^{-3}$ (the minimum BER that the forward error correction process can correct). While under the $4 \times 4$ MIMO scenario, we can achieve a SNR gain above $30 \mathrm{~dB}$ than ZF algorithm. And what's more, when SNR is $60 \mathrm{~dB}$, the BER will be greater than 0.1 and we can't demodulate the source information employing ZF algorithm. However, only about $10 \mathrm{~dB}$ of SNR is needed to achieve a $2 \times 10^{-3}$ BER employing the proposed SVD-based optical precoding scheme. The BER performances of the $2 \times 2$ MIMO and $4 \times 4$ MIMO systems almost keep the same with the channel correlation getting stronger, even under channels with strong correlation, the proposed scheme still has a good BER performance.

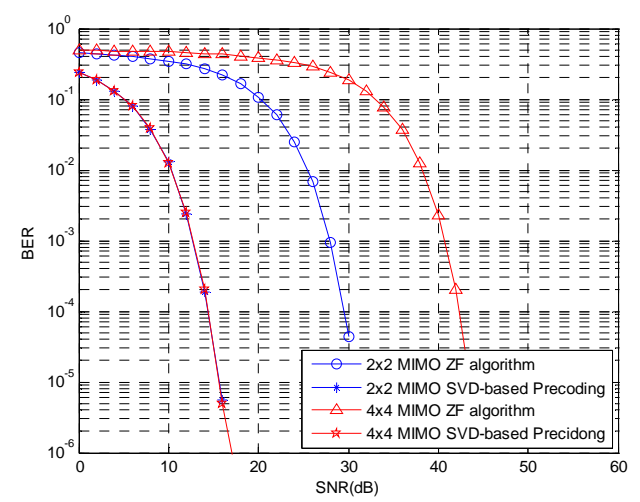

a. $d_{L E D}=0.8 m, d_{P D}=0.8 m$

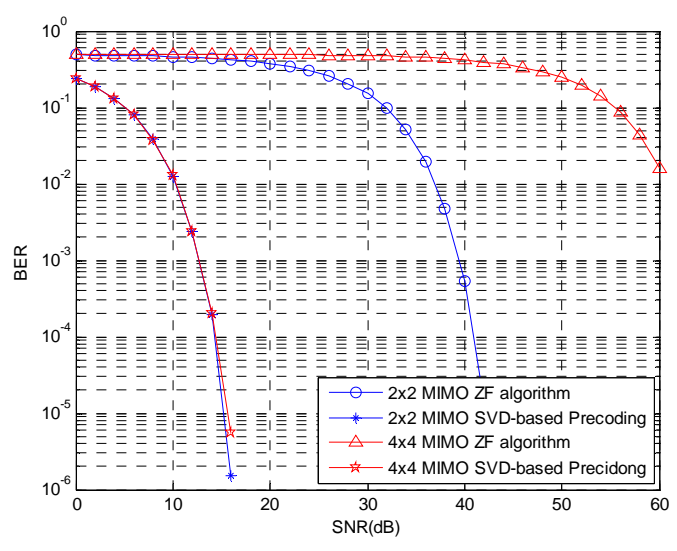

b. $d_{L E D}=0.4 m, d_{P D}=0.4 m$

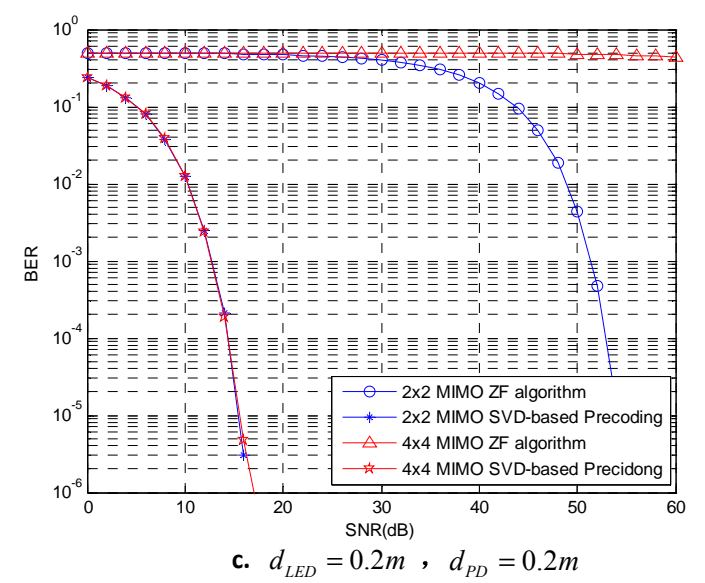

Fig. 5. Comparison of BER performance in MIMO system under different channel correlation.

According to the BER simulation of system under different channel correlation, it is observed that the BER performance of the proposed SVD-based optical precoding scheme can achieve a SNR gain more than $15 \mathrm{~dB}$ comparing to the ZF algorithm. This is because the proposed SVD-based optical precoding scheme can improve the SNR of the sub-channels with poor performance through effective power allocation and thus improving the BER performance of the overall system. Meanwhile, the BER performance of systems employing ZF algorithm decrease significantly as the channel 
correlation increases. When $d_{L E D}=0.2 \mathrm{~m}$ and $d_{P D}=0.2 \mathrm{~m}$, the $4 \times 4$ MIMO system employing $\mathrm{ZF}$ algorithm will be unable to demodulate the source information, while the BER performance of systems based on the proposed SVD-based Precoding almost keep the same with the channel correlation getting stronger, and high spatial multiplexing gain under strong correlation channels is conceivable.

\section{CONCLUSION}

In this paper, we propose an optical precoding scheme based on SVD to solve the problem that it is difficult to obtain high spatial multiplexing gain under the strong correlation MIMO channels in indoor VLC system. First, the information transmitted must be mapped from a specific symbols constellation to ensure that the signals are nonnegative after precoding. Then, we decompose the indoor VLC MIMO channels by SVD, which can obtain the independent sub-channels to eliminate inter-channel interference. Finally, we solve the optimization problem aiming at the minimum system BER and subjecting to the limited total power to get the optimal power allocation matrix and allocate the power among the sub-channels according to the matrix to reach the equal performance of each sub-channel, which can decrease the system BER. The receiver adopts Maximum Likelihood (ML) algorithm to decode the received symbols after shaping process, and recovers the information by inverse symbols mapping. The system BER simulation results indicate that the BER of proposed SVD-based optical precoding scheme is significantly lower than ZF algorithm under different correlation channel. And with the enhancement of channel correlation, the BER performance remains in a relatively low level, so it can obtain high spatial multiplexing gain under the strong correlation channels.

Indoor VLC system has great interior spatial resources. But the strong correlation channels make it difficult to achieve high spatial multiplexing gain, which becomes a limiting factor to increase the data transmission speed. The proposed SVD-based optical precoding scheme is just one way to achieve high spatial multiplexing gain. How to make better use of interior spatial resources in VLC system to obtain higher spatial multiplexing gain still needs further study.

\section{REFERENCES}

[1] H. Elgala, R. Mesleh, and H. Haas, "Indoor optical wireless communication: potential and state-of-the-art," IEEE Communications Magazine, vol. 49, no. 9, pp. 56-62, Sep. 2011.

[2] M. Saadi, L. Wattisuttikulkij, Y. Zhao et al., "Visible light communication: opportunities, challenges and channel models," International Journal of Electronics \& Informatics, vol. 2, no. 1, pp. 1-11, Feb. 2013

[3] S. Schmid, G. Corbellini, S. Mangold et al., "LED-to-LED visible light communication networks," in Proc. the fourteenth ACM International Symposium on Mobile Ad Hoc Networking and Computing, Bangalore, 2013, pp. 1-10.

[4] J. Vucic and K. D. Langer, "High-speed visible light communications: State-of-the-art," in Proc. IEEE Optical Fiber Communication
Conference and Exposition, and the National Fiber Optic Engineers Conference, Los Angeles, 2012, pp. 1-3.

[5] T. Q. Wang, Y. A. Sekercioglu, and J. Armstrong, "Analysis of an optical wireless receiver using a hemispherical lens with application in MIMO visible light communications," IEEE Journal of Lightwave Technology, vol. 31, no. 11, pp. 1744-1754, June 2013.

[6] M. D. Renzo, H. Haas, and P. M. Grant, "Spatial modulation for multiple-antenna wireless systems: a survey," IEEE Communications Magazine, vol. 49, no. 12, pp. 182-191, Dec. 2011.

[7] S. G. Wilson, M. B. Pearce, and Q. Cao et al., "Free-space optical MIMO transmission with q-ary PPM," IEEE Transactions on Communications, vol. 53, no. 8, pp. 1402-1412, Aug. 2005.

[8] D. O'Brien, "Multi-input multi-output (MIMO) indoor optical wireless communications," in Proc. the Forty-Third IEEE Asilomar Conference on Signals, Systems and Computers, Pacific Grove, 2009, pp. 1636-1639.

[9] L. Zeng, D. O'Brien, H. Minh et al., "High data rate multiple input multiple output (MIMO) optical wireless communications using white LED lighting," IEEE Journal on Selected Areas in Communications, vol. 27, no. 9, pp. 1654-1662, September 2009.

[10] D. O'Brien, "Optical multi-input multi-output systems for short-range free-space data transmission," in Proc. IEEE International Symposium on Communication Systems Networks and Digital Signal Processing, Newcastle, 2010, pp. 517-521.

[11] T. Fath and H. Haas, "Performance comparison of MIMO techniques for optical wireless communications in indoor environments," IEEE Transactions on Communications, vol. 61, no. 2, pp. 733-742, Feb. 2013.

[12] L. Wu, Z. C. Zhang, and H. P. Liu, "Modulation scheme based on precoder matrix for MIMO optical wireless communication systems," IEEE Communications Letters, vol. 16, no. 9, pp. 1516-1519, September 2012

[13] T. Komie, "Fundamental analysis for visible light communication system using LED lights," IEEE Transactions on Consumer Electronics, vol. 50, no. 1, pp. 100-107, Feb. 2004.

[14] T. A. Tran and D. O'Brien, "Performance metrics for multi-input multi-output (MIMO) visible light communications," in Proc. IEEE International Symposium on Communication Systems Networks and Digital Signal Processing, Pisa, 2012, pp. 1-3.

[15] L. Zeng, D. O'Brien, H. L. Minh et al., "Improvement of date rate by using equalization in an indoor visible light communication system," in Proc. IEEE International Conference on Circuits and Systems for Communications, Shanghai, 2008, pp. 678-682.

Qing-Feng Liu is currently an associate professor and a supervisor of postgraduate Student. Now, he is the assistant director of Mobile Communication Department for National Digital Switching System Engineering \& Technological R\&D Center. His research interests include wireless mobile communication network and information secrecy.

Shuai-Fang Xiao received his B.E. degree in electronic engineering Department from Tsinghua University. He is currently a M.S. candidate at National Digital Switching System Engineering \& Technological Research Center. His research interests are mobile communication, signal processing and visible light communication.

Kai-Zhi Huang was born in 1973, who received the Ph.D. degree in communication and information system from Tsinghua University. She is currently a professor and supervisor of postgraduate students. Now, she is the assistant director of Mobile Communication department for National Digital Switching System Engineering \&Technological R\&D Center. Dr. Huang is serving as a leader of Henan "wireless mobile communication innovation technology team" and "excellent technology innovation group of General Staff Headquarters". Her research interests include wireless mobile communication network and information secrecy.

Zhou Zhong received his B.E. and M.S. degrees in 2005 and 2008 respectively, from the Department of Communication Engineering, Zhengzhou Information and Science Institute. Currently, he is a Ph.D. candidate at the National Digital Switching System Engineering \& Technological Research Center. His research interests lie in the field of coding theory, signal processing and physical layer security in wireless communication. 